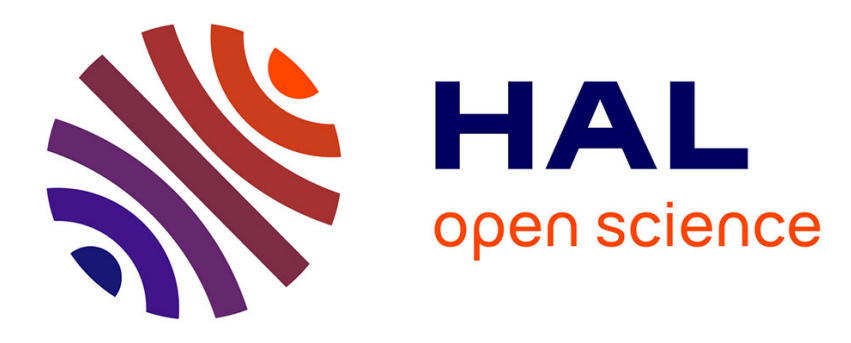

\title{
La dame courtoise et la littérature dans Les Miracles de Nostre Dame de Gautier de Coinci
}

\author{
Jean-Louis Benoit
}

\section{To cite this version:}

Jean-Louis Benoit. La dame courtoise et la littérature dans Les Miracles de Nostre Dame de Gautier de Coinci. Le Moyen Age. Revue d'histoire et de philologie, 2010, 2 (CXVI), pp.367-384. hal-00941488

\author{
HAL Id: hal-00941488 \\ https://hal.science/hal-00941488
}

Submitted on 3 Feb 2014

HAL is a multi-disciplinary open access archive for the deposit and dissemination of scientific research documents, whether they are published or not. The documents may come from teaching and research institutions in France or abroad, or from public or private research centers.
L'archive ouverte pluridisciplinaire HAL, est destinée au dépôt et à la diffusion de documents scientifiques de niveau recherche, publiés ou non, émanant des établissements d'enseignement et de recherche français ou étrangers, des laboratoires publics ou privés. 


\section{La dame courtoise et la littérature dans Les Miracles de Nostre Dame de Gautier de Coinci}

Une des caractéristiques de l'idéologie et de la littérature courtoises est la place éminente accordée à la femme. Celle-ci est une dame de haute naissance, souvent mariée. Il est inutile de rappeler ici la doctrine de la fin'amor qui s'est constituée autour du service qui lui est dû ${ }^{1}$. Tout un code règle le comportement de l'amant qui sert et vénère sa dame. A des degrés divers, cette éthique et cette esthétique sont présentes dans la majeure partie de la littérature médiévale, en particulier la lyrique et le roman ${ }^{2}$. Pour peu que l'on veuille faire de la littérature, désormais il faut s'y référer. Le public, aristocratique ou non, a ses goûts et ses attentes. Les aventures chevaleresques gagneront à être aussi amoureuses. La poésie chante surtout la dame aimée. L'Église ne pouvait ignorer ce domaine de la culture qui lui échappait de plus en plus. Notre hypothèse est que les Miracles de Nostre Dame, qui fleurissent dans toute la Romania, à la fin du XII ${ }^{\mathrm{e}}$ siècle et au cours du XIII ${ }^{\mathrm{e}}$ siècle $^{3}$ sont une tentative pour proposer une littérature sacrée qui puisse rivaliser avec la littérature profane, dans laquelle triomphe la dame courtoise ${ }^{4}$. Ces miracles sont des traductions versifiées et souvent des réécritures des innombrables miracles latins qui célèbrent les merveilles de Notre-Dame. Gautier de Coinci est le plus célèbre de ces auteurs. Il a vécu dans la région de Soissons. Il était prieur de l'abbaye de Vic-sur-Aisne, puis de Saint Médard de Soissons. Il écrit ses miracles de 1218 à 1227 et jusqu'à sa mort en 1236, il compose chaque année au moins une chanson en l'honneur de la Vierge, qu'il intègre à son recueil. Nous nous baserons sur l'édition de V. F. Koenig ${ }^{5}$. Son œuvre très variée ${ }^{6}$ et très riche a eu un succès considérable au Moyen Age, comme l'attestent les nombreux manuscrits qui nous restent. C'est lui qui définit véritablement le miracle comme genre littéraire. Nous nous proposons d'observer comment cet auteur traite du thème de la courtoisie et plus particulièrement de la dame courtoise dans son œuvre. Cette question nous conduira à en examiner secondairement une autre, plus complexe, celle du rapport de la littérature mariale et de la littérature profane. La théorie qui prévaut est celle selon laquelle la littérature mariale ne fait que copier la littérature profane. Est-ce exact?

N'imaginons pas les auteurs de miracles comme des dénonciateurs intransigeants de la littérature courtoise. Ils la connaissent, ils savent son succès et pensent comme Adgar, un moine anglais du XII ${ }^{\mathrm{e}}$ siècle, précurseur de Gautier, qu'on peut en tirer quelque chose de bon (la courtoisie et la sagesse, mais aussi beaucoup d'autres vertus ${ }^{7}$ ) :

\footnotetext{
${ }^{1}$ Cf. R. BEZZOLA, Les Origines et la formation de la littérature courtoise en Occident, Paris, Champion, 19601963.

${ }^{2}$ Avec des nuances entre les deux, évidemment. Le service dû à la dame est parfois la prouesse dans le roman et la dame y est moins souvent une épouse adultère (Guenièvre reste toutefois un exemple majeur).

${ }^{3}$ P. BETEROUS (Les collections de miracles de la Vierge en gallo et ibéro-roman, au XIIIe siècle, Dayton, USA, 1983) propose la meilleure synthèse sur les diverses collections de miracles.

${ }^{4}$ Sur la constitution du genre des miracles, voir F. GINGRAS, Les miracles de Notre-Dame dans la typologie des genres narratifs des XII ${ }^{\mathrm{e}}$ et XIII ${ }^{\mathrm{e}}$ siècles, Mélanges offerts à Pierre Kuntsmann, Ottawa, éd. David, 2008.

${ }^{5}$ GAUTIER DE COINCI, Les Miracles de Nostre Dame, t. 1, 2, 3, 4, éd. V. F. KOENIG, Genève, Droz, $1966-$ 1970.

${ }^{6}$ Chaque miracle comporte un récit de faits miraculeux et un abondant commentaire personnel de l'auteur. Le recueil contient aussi deux longs sermons (De la misere d'ome et de fame, De la chasteé as nonains) et de multiples chansons à Notre-Dame.

${ }^{7}$ Cf. M. LAZAR, Amour courtois et fin'amors dans la littérature du XIIe siècle, éd. Klincksieck, 1964, p. 28-46.
} 


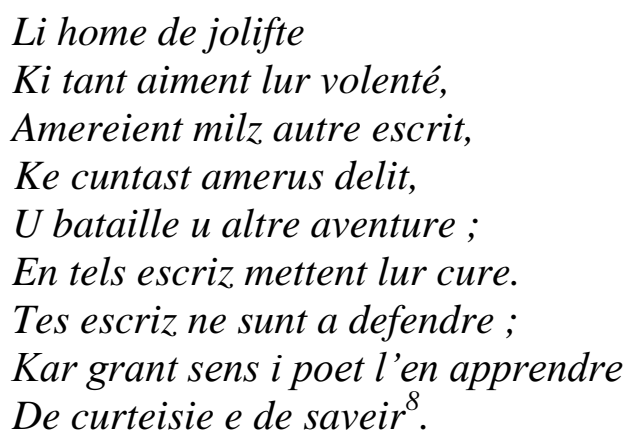

Il y a cependant, dans les miracles, une condamnation très nette de la passion amoureuse vouée à une dame, selon la doctrine de la fin'amor. Un des miracles les plus représentatifs à cet égard est De un chevalier (t.3, p.150). Gautier de Coinci nous présente un chevalier :

Josnes, biaus, cointes, fors et fiers,

De grant affaire et de grant non. (v.2-3)

Il n'est intéressé que par les fêtes et les tournois. Il se prend de passion pour une dame. Pour elle, il multiplie les tournois et dépense beaucoup d'argent. Il refuse de chercher une femme avec qui il puisse se marier. C'est sans doute ce qui semble le plus grave aux yeux de l'auteur :

De tant ert solz et malaisins
Qu'esposer fame ne voloit,
Car li corages li bouloit
Si durement pour celle dame
Qu'il ne veoit a ses ielz fame
Qu'il daignast penre n'espouser

La dame pour laquelle il brûle jour et nuit est très belle mais fière et indifférente. Cette passion dévorante et sans issue est présentée, d'après une idée courante depuis Ovide, comme une grave maladie. Le saint abbé qu'il va consulter ne lui fait pas la morale, cela ne servirait à rien :

\section{Li preudom ne l'ose choser, Car il seit bien qui de tel chose \\ Si faites gens chastie et chose \\ Tant plus les esprend et atise.}

Il lui conseille seulement de dire chaque jour trois chapelets, soit cent cinquante « Je vous salue », ce que le jeune chevalier accepte de faire et qui le guérira de cette maladie. Tel est bien souvent l'amour, dans Les Miracles de Nostre Dame, une passion irrésistible et destructrice, qui détourne les laïcs du devoir de mariage et d'un amour réel. La dame, objet de cet amour ne peut que tromper les attentes de cet amant. Ici, elle rejoint la figure, bien représentée dans la lyrique amoureuse, de la cruelle indifférente, de la belle dame sans merci:

Tant la trova de grant fierté

C'onques de li ne peut avoir,

Por prïere ne por avoir

Ne por belle chevalerie,

Solas d'amor ne druërie;

Que plus la prie, plus est roide,

Et quant il plus la trueve froide,

Tant est il plus boulans et chaus. (t .3, p. 151, v. 40-47)

Les miracles nous offrent plusieurs cas de passion amoureuse dévastatrice. Notons d'ailleurs que l'auteur les décrit avec un réalisme, un sens psychologique, dignes des plus grands romanciers. Son intention est, bien entendu, de la condamner, comme immorale.

\footnotetext{
${ }^{8}$ ADGAR, Le Gracial, éd. P. KUNTSMANN, université d'Ottawa, 1982, p. 122, miracle XVI, v. 1-9.
} 
La beauté et la noblesse de la dame, toujours évoquées, déclenchent un amour coupable. Deux miracles nous présentent des situations à peu près analogues. Il s'agit cette fois d'une religieuse très belle et très noble qui se prend de passion pour un jeune chevalier beau et courtois. On trouve tous les éléments de l'aventure amoureuse. Il évoque dans D'une nonain qui vaut péchier, mais Nostre Dame l'en délivra (t. 2 p. 246), une religieuse très belle, de haute origine. Elle suscite l'amour d'un beau chevalier :

Un haut home de la contrée

De sa biauté si enflamma

Que si desveement l'ama

Por un petit n'issoit dou senz. (v. 18-21)

Celui-ci lui propose de partir avec lui :

Si seroit s'amie et sa dame. (v. 34)

Heureusement la Vierge intervient, dans un rêve, pour montrer à la religieuse la menace de l'enfer. Cela suffit à la faire changer d'avis.

Même scénario dans De la nonain (t. 3, p. 191). Même insistance du narrateur sur les qualités exceptionnelles de l'héroïne courtoise :

Pucelle estoit de haut linage,

De grant sens et de grant biauté.

Ne cuit qu'en une royaulté

Eüst plus bele damoysele.

S'ele ert de cors et de vis bele,

Encor ert plus bele de cuer. (v. 14-19)

Un neveu de l'abbesse (qui, au grand scandale de Gautier, loge dans son abbaye des membres de sa famille) tombe amoureux d'elle. Lui aussi est présenté selon les conventions de cette littérature :

Un damoisel vaillant et preu

Josne, envoisié, mignot et gent,

Estrait et né de haute gent. (t. 3, p. 192, v. 46-48)

La religieuse finit par céder à ses demandes et accepte de quitter le couvent pour le rejoindre. Une première fois la statue de la Vierge, devant qui elle s'agenouille tous les jours, l'en empêche. Elle y parviendra ensuite et vivra plusieurs années avec le jeune homme jusqu'à ce que la Vierge vienne lui apparaître pour lui faire regretter son infidélité. La dame prend conscience qu'il lui faut expier sa faute et elle informe son mari qu'elle veut retourner dans les ordres. Celui-ci fait de même de son côté. En choisissant des religieuses comme héroïnes de ces aventures amoureuses, en les présentant comme de parfaites dames courtoises, en racontant leurs fautes et leur repentir, Gautier veut souligner le caractère pernicieux et immoral de la fin'amor qui leur est portée. La transgression de la règle monastique est à ses yeux la plus grande faute qui soit.

Réciproquement, on voit aussi plusieurs religieux qui sont tentés par l'amour d'une dame. Admirable peintre du désir et de l'amour, Gautier décrit leur tourment avec compassion mais porte un jugement sévère sur cet amour qui ne respecte pas les lois les plus sacrées. C'est le motif, par exemple, des miracles que l'on a rassemblés sous le titre du «fiancé de la Vierge ${ }^{9} »$. On en trouve deux illustrations : De l'enfant qui mist l'anel ou doit l'ymage (t.2, p.197) et D'un clerc (t.4, p.340). Un jeune clerc a promis fidélité à la Vierge, en se consacrant à Dieu. Il oublie sa promesse et veut se marier. La Vierge intervient pour lui rappeler ses vœux. Là encore la demoiselle est très belle et très désirable :

Li clers, qui mise eut grant entente

En amer la gente pucelle,

\footnotetext{
${ }^{9}$ Cf. J. MORAWSKI, A. WYREMBEK, Les Légendes du fiancé de la Vierge dans la littérature médiévale, Poznan, 1934.
} 
Qui mout estoit mignote et bele,

Mout desira l'aler gesir

Por acomplir son grant desir. (t. 2, p. 200-201, v. 100-104)

Dans D'un clerc, Gautier pousse encore plus loin l'évocation. Le jeune clerc qui a accepté de se marier se retrouve à coté de sa charmante épouse sur le lit nuptial. L'auteur se plaît à décrire la beauté de la jeune fille et le désir du clerc en une scène très sensuelle. Il saura résister grâce à l'aide de Marie à cette ultime tentation :

Et li clers gist les la pucelle,

Qui si tres bele est come cele

Qui nature a faite a devise.

D'eures a autres mout l'avise,

Car mout li siet et mout li plaist . (t. 4, p.354, v. 373-377)

La passion amoureuse est souvent une occasion de pécher dans les miracles. Elle entraîne aussi bien les hommes que les femmes à toute sorte de folies ${ }^{10}$, la pire d'entre elles étant la rupture des vœux des consacrés. Tous les motifs de la peinture courtoise de l'amour y sont repris avec talent pour y être fermement dénoncés : beauté exceptionnelle de la dame, noblesse, renommée, coup de foudre, caractère irrésistible de la passion, transgression morale.

A vrai dire, la position de Gautier est nuancée. S'il est sans complaisance pour les péchés qui résultent de ces amours tumultueuses, s'il condamne la dame faible et coquette qui suscite l'amour ou qui y succombe elle-même, il essaie de définir une courtoisie véritable, idéale, qui conserve tout ce qu'elle a de positif, débarrassée des péchés qu'elle occasionne. On a, dans les miracles, une héroïne qui incarne parfaitement cet idéal. Il s'agit de l'impératrice du miracle De l'empereeis qui garda sa chasteé contre mout de temptations. La femme de l'empereur de Rome n'a que des qualités :

\section{L'empereeis, la damoysele, \\ Par defors fu plaisans et bele \\ De cors, de bras, de mains, de vis ; \\ Et se par dedens vos devis \\ La biauté de la bele dame \\ Plus que de cors bele fu d'ame.}

(t.3, p.305, v. 49-54)

Elle est le véritable modèle de la dame courtoise, car la beauté du corps et celle de l'âme sont associées. Le narrateur souligne ses qualités morales qui l'élèvent au-dessus de toutes les dames de la cour :

\section{Car tant est franche et debonaire}

Saige, cortoise et bien aprise

Que renommee plus la prise

Que toutes celles de l'empire. (t.3, p. 309, v. 154-157)

Au départ de son mari, elle est l'objet d'un amour passionné de la part de son beaufrère. L'auteur esquisse le scénario de ce qui aurait pu être le scénario d'une idylle courtoise entre elle et ce jeune seigneur, qui est aussi très séduisant, si le Diable ne s'en était mêlé :

\section{Li vallés ert de tel biauté \\ Ne cuit qu'ere une royauté \\ Plus bel ne plus plaisant eüst \\ S'anemis ne le deceüst.

Il lui fait une cour pressante. Les ravages de l'amour dans son cœur sont décrits avec autant de précision et de détails que dans les meilleurs romans. Les dialogues et les descriptions retracent cette tentative de séduction et les résistances polies de l'impératrice. Celle-ci est aussi délicate que ferme. Elle a même pitié de son soupirant (v.319). Cependant,

\footnotetext{
${ }^{10}$ Les poètes ne cessent ne célébrer la folie de l'amour.
} 
contrairement à d'autres, elle reste fidèle à son mari, éconduit son prétendant trop entreprenant et finit par l'enfermer dans une tour. Elle devient alors une véritable martyre de la fidélité et de la chasteté, car, au retour de son mari, le monstrueux beau-frère, poussé par la rage et le dépit, l'accusera de débauche et d'avoir voulu le séduire. L'empereur le croit et bannit son épouse, avec une odieuse brutalité. Commenceront alors une série de malheurs où resplendiront toujours mieux la fidélité, la pureté, la piété, la charité de l'impératrice (elle pardonnera même à ses bourreaux). Notons que ces péripéties tragiques fournissent la trame d'excellentes aventures romanesques, pathétiques à souhait, couronnées par l'intervention merveilleuse de la Vierge. La démonstration est claire. La courtoisie sous sa forme la plus haute est compatible avec la fidélité et le mariage. Gautier défend avec ferveur le mariage, surtout auprès de :

Ces grans signeurs et ces grans dames

Qui por les cors perdent les ames. (t. 3, p. 304, v. 29-30)

Il s'appuie sur les Écritures et la tradition de l'Église. Il va jusqu'à écrire :

Qui tient mariage a eschar

Et qui desront et devise

Dieu escharnist et Sainte Eglise. (t. 3, p. 306, v. 86-88)

Il met en garde contre les beaux parleurs qui savent chanter les louanges de celle qu'ils veulent séduire. C'est un véritable poème d'amour qu'il met dans la bouche du perfide beaufrère (t. 3, p. 314-315, v. 289-315). Celui-ci cite même avec à-propos des références littéraires pour convaincre sa belle (Tristan et Iseut, Pyrame et Thisbé).

Si le mariage est sincèrement réhabilité, il n'en reste pas moins que l'union la plus élevée est, pour Gautier, l'union avec Dieu dans la vie consacrée ${ }^{11}$. L'impératrice, d'ailleurs, rentrera dans les ordres à la fin de l'histoire. D'autres religieuses sont proposées en modèle dans les miracles. L'auteur souligne leurs qualités en des termes courtois. Dans le sermon De la chasteé as nonnains (t. 3, p. 463), il s'adresse aux religieuses de Soissons. Elles aussi sont des jeunes filles belles, nobles et vertueuses. Elles ont donné toute leur vie pour le seul amour parfait, pour le Christ. En des termes propres à tous les mystiques, il évoque le bonheur céleste que leur accordera le Divin Époux. Il se plaît par exemple à détailler les beaux vêtements que revêtiront leurs âmes, alors qu'elles ont tout sacrifié en cette vie :

Vos indes fleurs, vos vïoletes,

Qui les grans plices d'erminetes,

Qui la soie, le vair, le gris

Avez laissiez pour les dras bis,

Qui pour les ames faire blanches

Vestez les fros as noires manches

Sachiez que Diex em paradys

De vos fera ses flors de lis.

Vos blanches fleurs, vos de Cistiaus,

Qui afubleez as blans mantiaus,

Qui les pliçons, qui les chemisez

Pour les blans buiriax avez jus misez

Ja sont ou ciel aparilliez

Blanches chemises deliees

Et les robes a or batues

Dont vos ames seront vestues. (t. 3, p. 500-501, v. 1059-1074)

\footnotetext{
${ }^{11}$ Cf. A. GARNIER, Le Miracle de la chaste impératrice, Paris, Honoré Champion, 2007, p. 33. Mutations temporelles et cheminement spirituel, analyse du miracle de l'empereeis de Gautier de Coinci, Paris, Honoré Champion, 1987.
} 
Il développe alors un thème qui lui est cher, prenant le contre-pied d'une formule courtoise éculée sur l'amertume de l'amour: En vostre ami n'a point d'amer (p. 502, v. 1110). Il s'amuse même à proposer à ces religieuses, qui ont une jolie voix, de chanter une chanson, non pas de mal mariée, mais de bien mariée. Il en fournit une sur le modèle de ces contrafactures dont il a le secret. En voici le début :

\section{«La fontenele i sort clere}

Bonne aventure ait ma mere,

Qui si bien me maria!» (t. 3, p. 503, v. 1117-1119)

En effet, c'est Jésus-Christ, fils de Marie, qui a pris le cœur de chacune d'elles. Il ne peut que leur réserver un bonheur éternel et leur éviter les déboires de tous ces amants infidèles et inconstants.

L'ami de cœur n'est pas nécessairement le Divin Époux des âmes. Gautier envisage dans le miracle De l'ymage Nostre Dame de Sardanei (t. 4, p. 378), une sainte amitié entre un moine de Constantinople et la religieuse qui a établi un ermitage près de Damas, à Sardanei, où elle prie Dieu et Notre-Dame. Elle voudrait avoir une image de la Vierge pour mieux la vénérer. Le moine, qui part en pèlerinage à Jérusalem, lui propose d'en rapporter une pour elle. Après bien des tergiversations, des aventures et des prodiges, le moine, sous la pression de Dieu, se décide enfin à rapporter cette icône qui s'avèrera miraculeuse. C'est le couronnement d'une belle histoire. La religieuse se réjouit autant d'avoir rencontré un compagnon pour servir Marie que d'avoir l'icône. Le terme qu'elle emploie joie parfaite appartient au lexique courtois :

\section{Or est ma joie bien parfaite \\ Fait la none, bialz doz amis \\ Quant compaignon m'a Diex tramis \\ Mesmement moigne et provoire. (t. 4, p. 392, v. 374-377)}

On trouve d'ailleurs toutes les péripéties de ce type de récit: la rencontre, le service, la séparation, les retrouvailles, l'union, la joie. Les dialogues sont marqués par une grande tendresse, sans aucune ambiguïté. Le modèle narratif est celui d'une quête faite par le héros. Quête d'un objet sacré au bénéfice de la dame qui en fait la demande. On le voit, les échos de ce type d'intrigue sont nombreux dans la littérature médiévale. Une fois de plus, Gautier adapte à son sujet les structures et les thèmes de la littérature courtoise. Avec audace, il affirme la possibilité d'une profonde amitié, pourvu qu'elle soit sainte, entre un homme et une femme. Leur union, au service de Dieu et de sa mère, dégage un doux parfum :

A sobrement et a bien vivre

Andui si misent leur entente

Que plus que mugés ne que mente

Flaira soef leur renomee. (t. 4, p. 392, v. 388-391)

La dame courtoise par excellence, dans les miracles, est évidemment Notre-Dame :

Nostre Dame Sainte Marie

Qui fontaine est de cortoysie. (t. 4, p. 177, v. 49-50)

Elle est une reine, la Reine du Ciel et de la Terre :

En Paradys siet a la destre

De son doz fil, le roy celestre.

La est de toz ses sainz servie

Et hounouree et conjoïe,

D'angeles, d'archangeles encenssee. (t. 4, p. 536, v. 2463-2467)

Le motif courtois du service dû à la dame est aussitôt posé. Elle n'a pas besoin qu'on la serve, pourtant il faut le faire. Elle y consent par intérêt pour nos âmes :

Non pas pour ce que Nostre Dame 
Nul mestier ait de no service,

Mais tant est plainne de franchise

Que tous jors vielt que la servons

Tant que s'ä̈e deservons. (t. 4, p. 536, v. 2450-2454)

L'aimer et la servir c'est être assuré d'être aimé définitivement, éternellement, en retour. Cet amour réciproque est donc la source d'un vrai bonheur pour le cœur et pour l'âme. Cette fois encore «l'amour amer», avec ses souffrances et ses désillusions, est évité :

En si douce dame amer

A grant deduit

En s'amor n'a point d'amer

Ançois i a grant fruit. (t.1, p.39, v.49-52)

Le poète peut chanter sur tous les tons la joie qu'il y a de se marier à Marie :

Marions nous a la Virge Marie

Nus ne se puet en li mesmarier.

Sachiez de voir, a li qui se marie

Plus hautement ne se puet marïer. (t.1, p.30, v. 28-31).

Tous ses amans met ou ciel et marie.

Mout se fait bon marier a Marie. (t.1, p.47, v.7-8)

Il est vrai qu'elle n'a pas de ces terribles préférences qui mettent à l'écart des amants jugés indignes. Elle aime également les beaux et les laids, les bons et les mauvais, les bien-portants et les malades. Elle a même une prédilection pour ceux qui souffrent et qui sont rejetés. En effet, elle aime tout particulièrement les malades, les pauvres et les pécheurs. Elle attend de chacun un simple élan d'amour pour y répondre avec empressement. Il faut que cet élan soit sans réticence, ni tromperie, de la part de celui qui s'y engage :

Qui la pucele vielt amer

En qui amor n'a point d'amer

Amer la doit de tel corage

Qu'il n'ait le cuer frans ne volage.

Ele par est si vraie amie

Que rien ne sait de doublerie. (t.4, p.441, v.43-48)

Les clercs et les prêtres en particulier doivent la préférer à toute femme :

Sache, sache qui s'entremet

D'acointier si tres vraie amie

Com ma dame Sainte Marie,

Mout vrai amans li couvient estre

Amons la tuit, et clerc et prestre. (t. 4, p. 441-442, v. 62-66)

En effet quelques miracles nous présentent le cas d'un clerc qui préfère choisir une épouse plutôt que de rester fidèle à la promesse qu'il a faite de servir Notre-Dame, de se consacrer à elle et de l'aimer fidèlement. On assiste alors à la scène étonnante de l'apparition de la Vierge qui vient faire de sérieuses remontrances à ce clerc infidèle un peu sur le ton d'une épouse jalouse, par exemple dans le miracle d'un clerc où un jeune clerc voulait se marier (t.4, p.351352, v. 284-312). Nous l'avons dit, le miracle le plus représentatif du motif du « fiancé de la Vierge » est, dans l'œuvre de Gautier, De l'enfant qui mist l'anel ou doit l'ymage. On y voit un jeune homme qui a reçu de son amie un anneau. Il est émerveillé par la beauté de la statue de Marie. Il décide de mettre alors cet anneau au doigt de la statue en signe de son engagement :

"Cest anel ci qui mout est biau,

Te veil doner par fine amor... »

( t.2, p.199, v.54-55) 
La statue plie alors la main en signe d'acquiescement. Le jeune homme oublie sa promesse et va se marier. La Vierge lui apparaît dans une vision et lui rappelle sa promesse de parfait amour. Le jeune homme renonce au monde pour se consacrer à la vie religieuse. Joseph Morawski démontre que l'origine de ce miracle est une légende païenne. C'est Vénus qui veut se venger de l'infidélité de celui qui avait promis de s'unir à elle, avant de rompre ses vœux pour une femme. Il y a quelque risque à transposer, même par souci d'édification, une légende aussi sulfureuse dans le domaine chrétien (ce sont les devanciers de Gautier qui l'ont fait en latin). Marie risque de garder des caractéristiques inopportunes de cette sourcilleuse déesse $^{12}$. On retrouve ce même danger dans quelques miracles où Marie ressemble d'un peu trop près à une héroïne de roman. On la voit par exemple «échevelée », en chemise, venir défendre contre des animaux sauvages (le Diable, en vérité) un moine ivre. La «belle demoiselle $»$ a quelque chose d'une fée ${ }^{13}$.

D'une manière générale, le poète n'a pas assez de mots pour célébrer la perfection de Marie. Sa beauté, sa bonté, sa noblesse, sont inlassablement exaltées. Elle est la seule dame courtoise vraiment parfaite :

\section{Tu as biauté \\ Et loiauté \\ Valor et cortoisie (t.1, p. 34, v. 62-64).}

Plus d'une fois l'auteur prend plaisir à la décrire dans l'éclat de son humanité et de sa féminité, elle en qui rejaillit la lumière du Ciel. Retenons par exemple cette belle description de l'apparition de Marie au sacristain très pieux qui avait demandé la grâce de voir Notre Dame. Celle-ci lui apparaît pendant son sommeil :

Au moigne, luez qu'endormis fu,

Sambla por voir que plains de fu

Fust li mostiers et plains de flamme,

Car devant lui vint une dame

Qui fu plus clere que solaus

A mïedi quant est plus haus.

Et fu d'une robe vestue

Qui toute fu a or batue,

Plaine de pierres precieuses

Si cleres et si glorïeuses

Toz li mostiers resplendissoit

De la lueur qui en issoit.

Plus avoit blonz les crins et sors

Et plus luisanz que n'est fins ors,

Et si tres clers si oel estoient

Que deus estoiles resambloient.

Resplendissant avoit la face

Plus qu'esmeraude ne thopace.

Une couleur avoit rosine

Si tres esmeree et si fine,

Si deliteuse et si tres bele

Rien n'i fesist rose novele. (t. 3, p. 14-15, v. 79-100)

La dame n'est pas seulement merveilleusement belle. Elle ne se contente pas de représenter un idéal féminin décrit avec la rhétorique courtoise. Elle est mère de Dieu et messagère céleste. Elle se présente au moine comme une éducatrice, avec un livre magnifique, orné d'or

\footnotetext{
${ }^{12}$ J. MORAWSKI, op. cit., p. 16. Il considère même que cette substitution a quelque chose de « grotesque » (p. 17)

${ }^{13}$ De un moigne que Nostre Dame delivra dou Dyable, t. 2, p. 115.
} 
et de vermillon, qui n'est autre que le livre d'Isaïe, où, on le sait, sont annoncés la venue du Messie et le rôle de la Vierge. Celle-ci va faire davantage encore pour lui. Alors qu'il ose lui demander l'autorisation d'embrasser ses pieds, elle se penche tendrement et maternellement et lui laisse embrasser son visage :

\section{Nostre Dame sainte Marie \\ Sousriant dist : "Je ne veil mie, \\ Biaus doz amis, que je n'atouche \\ A mes piez ta sainte de bouche, \\ Qui tantes fois m' a saluee. \\ Mais en ma face coloree, \\ Biaus doz amis, me siet et plaist}

Que ta bele bouche me baist. » (t. 3, p. 17, v. 162-168)

Dans plusieurs autres miracles, la Dame du Ciel, loin d'être une créature désincarnée, se penche avec tendresse et amour sur le corps blessé de tant de malades qu'elle n'hésite pas, pour les guérir, à caresser, à arroser même de son lait (D'un moigne, t. 3, p.138-139, v.118129). En cela elle se distingue des grandes dames de ce monde, facilement hautaines, méprisantes et vite dégoûtées par les misères et les faiblesses des hommes :

Comment est ce ne dont avient

Qu'a la dame dou ciel sovient

De tel merdaille com nous sommes,

Qui d'ors pechiez portons granz sommes? (t. 4, p. 537, v. 2475-2478)

Elle a aussi la supériorité évidente sur les dames de la Terre de ne pas être soumise à l'usure du temps et à la fatalité de la mort. Le poète retrouve le ton moraliste d'Hélinand de Froidmont, auteur des Vers de la mort, pour rappeler ce topos de la prédication :

\section{Fi! fi!fi! fi! Que vaut hautece}

Que vaut honneurs? Que vaut richece?

Que vaut au roy sa royautez?

A royne que vaut biautez? (t. 4, p. 519, v.2047-250)

On le voit, Gautier, à travers l'image de la dame, nous présente une image mitigée de la courtoisie. Il condamne sans appel la vision de l'amour qu'elle véhicule, mais essaie d'en conserver les valeurs. Il les transpose dans le domaine religieux. Plusieurs figures de la dame courtoise, dans les miracles, tendent à prouver qu'il est possible de concilier ces valeurs humaines et sociales avec les lois divines, d'abord dans le mariage. Il présente surtout la Vierge Marie comme l'idéal féminin de la dame courtoise. Chacun est invité à la servir. Ce service est un véritable hommage. Il est attendu du héros, du lecteur, de l'auteur lui-même. Curieusement, Jésus, qui est Dieu, au Jugement des âmes, semble à plusieurs reprises servir sa mère, la dame du Ciel, et lui accorder le salut pour tous les pécheurs qu'elle veut sauver. On trouve dans les miracles tous les motifs attachés à cette littérature. Il pourrait être tentant, alors, de faire de la littérature mariale un pur décalque sans intérêt de la littérature courtoise. La critique s'est concentrée sur la poésie. C'est l'opinion de Pierre Bec qui écrit : "Tant pour ce qui et du contenu que de la forme, ce lyrisme a littéralement calqué, avec plus ou moins de bonheur et de servilité, la thématique et l'écriture propres au lyrisme profane. Plus que d'interférences registrales, on pourrait presque parler ici de registre parasite ${ }^{14}$. » Incontestablement, comme l'écrit Gérard Gros «Au premier quart du XIII ${ }^{\mathrm{e}}$ siècle apparaît une catégorie nouvelle de chanson, la chanson pieuse, ou chanson mariale. Il semble bien que l'initiateur de cette variante du genre soit, à partir de 1220, un moine bénédictin de Soissons, excellent poète : Gautier de Coinci (vers 1177-1236). De par son éducation, ce trouvère pieux

\footnotetext{
${ }^{14} \mathrm{P}$. BEC, La Lyrique française au Moyen Age (XII ${ }^{e}$ XIII ${ }^{e}$ siècles). Contribution à une typologie des genres poétiques médiévaux, Etudes et textes, t. 1, Paris, 1977, p. 142.
} 
connaissait bien la doctrine courtoise : installée en Champagne (le terroir septentrional de la courtoisie) sa famille entretient des relations avec l'aristocratie.

La chanson ainsi réorientée par le culte marial (alors florissant) est un contrafactum- en langue romane une contrafacture- c'est à dire, en français moderne, une imitation : il s'agit de célébrer la Vierge en employant une mélodie de chanson profane ${ }^{15}$. Ce qu'on voit naître est un cantique enjolivé de grâce courtoise ${ }^{16}$. » Gérard Gros souligne ailleurs "l'embarrassant problème du transfert de l'hommage courtois à la figure de Notre-Dame ${ }^{17}$ ». Ces problèmes sont comparables à ceux que nous avons évoqués pour les miracles narratifs à propos du motif du fiancé de la Vierge. Faut-il distinguer la poésie et les miracles narratifs ? Certes les genres sont différents, mais Gautier a voulu que son œuvre constituât un tout. Elle comprend trois volets: les miracles, les chansons et les deux sermons. Ces chansons, il les dispose volontairement, comme des «fleurettes», entre les miracles. Chansons et miracles sont animés de la même ferveur mariale, du même souci d'édification. La personne de Marie en constitue le centre et l'unité. La poésie déborde souvent même du cadre narratif du miracle en de fascinantes prouesses rhétoriques. Le lyrisme, le recours à l'exemplum narratif et la réflexion théologique se répondent constamment. Certes, les chansons reprennent incontestablement formes et motifs de la chanson profane, mais dans quelle intention ? Avec un sens de la formule, Jean Frappier parle «d'une tentative de conversion » de la lyrique profane amoureuse ${ }^{18}$. Anna Drzewicka évoque un « essai d'évangélisation de la culture profane ou [un] phénomène d'inculturation : le message religieux de l'Eglise adoptant [...] le langage propre à une civilisation ${ }^{19}$ ». Jacques Chailley, lui aussi, démontre que Gautier est l'initiateur du principe du contrafactum dans la littérature musicale mariale en langue vulgaire. Mais, selon cet illustre musicologue, Gautier aurait puisé son inspiration surtout dans la lyrique religieuse latine : «La lyrique latine, parfois profane, mais restant le plus souvent d'inspiration religieuse, était en plein essor à l'époque où écrivait le prieur de Vic. Saint Martial de Limoges était passé un peu à l'arrière-plan mais l'école de Notre-Dame de Paris et celle de Saint-Victor rédigeaient alors leurs grands manuscrits ${ }^{20}$. » Selon lui, en général, Gautier puise à la fois à la source sacrée et à la source profane : "Les chansons de Gautier de Coinci paraissent ainsi un élément de liaison précieux entre la lyrique latine et celle des trouvères. A la première, il emprunte les thèmes favoris de louange mariale, avec plus de délicatesse que ses modèles [...] mais à cette source primitive, il mêle avec bonheur les images ou tournures gracieuses de modèles profanes, voire amoureuse, les incipits et refrains à peine travestis ${ }^{21}$. $» \mathrm{Au}$ niveau de la thématique, l'expression d'un amour absolu pour une dame que l'on va servir n'est pas une invention des troubadours. Adressée à la Vierge, cette dévotion lyrique n'est pas nouvelle : «L'expression de l'amour, de l'adoration et de la volonté de service est une démarche pour ainsi dire obligatoire dans toute littérature religieuse de teinte lyrique ${ }^{22}$, et on n'a pas attendu les modèles profanes fournis par les

\footnotetext{
${ }^{15}$ Cf. par exemple, J.H. MARSHALL, Gautier de Coinci imitateur de Guilhem de Cabestanh , Romania, 98, 1977 , p. 245-249.

${ }^{16}$ G. GROS, M. M. FRAGONARD, Les Formes poétiques du Moyen Age à la Renaissance, Paris, Nathan, 1995 , p. 26.

${ }^{17}$ G. GROS, Lyrisme médiéval (XII ${ }^{\mathrm{e}}$-XIII ${ }^{\mathrm{e}}$ siècles) poésie courtoise et poésie pieuse, Perspectives Médiévales, trente ans de recherche en langue et littérature médiévales, mars 2005, p. 236.

${ }_{18}^{18}$ J. FRAPPIER, La Poésie lyrique en France au XII et XIII $I^{e}$ siècles, Paris, 1960, p. 77.

${ }^{19}$ A. DRZEWICKA, La fonction des emprunts à la poésie profane dans les chansons mariales de Gautier de Coinci , Le Moyen Age, t. 91, 1985, p. 40.

${ }^{20}$ J. CHAILLEY, Les Chansons à la Vierge de Gautier de Coinci, Paris, thèse dactylographiée, 1952, p. 109, Heugel, 1959.

${ }^{21}$ Ibidem, p. 65.

${ }^{22}$ Cf. E. PALAZZO, A.-K. JOHANSONN, Jalons liturgiques pour une histoire du culte de la Vierge dans l'Occident latin ( $\mathrm{V}^{\mathrm{e}}-\mathrm{XI}^{\mathrm{e}}$ siècles), Le culte de la Vierge dans la société médiévale, éd. Beauchesne, 1996.
} 
troubadours et les trouvères pour en parler $^{23}$. » On la trouve, par exemple, dans la tradition hymnologique et liturgique latines. Il est donc possible, à l'inverse, de considérer que la thématique amoureuse profane a beaucoup emprunté au culte marial. Pierre-Yves Badel le pressent en écrivant : «... La littérature de cour exalte un idéal tout profane (la courtoisie, l'amour), mais elle ne peut la formuler qu'en recourant au vocabulaire religieux et en concevant la relation de l'homme et de la femme sur le modèle de l'homme à Dieu ${ }^{24}$. » De nombreux critiques ont noté que l'idéal courtois de la dame et le culte qui lui est rendu ont une dimension religieuse. La fin'amor n'est-elle pas une «religion de l'amour», pour reprendre une formule célèbre ? Yves Lefèvre, par exemple, démontre que le premier des troubadours, Guillaume IX, adresse un culte à la dame sur le modèle du service religieux, pour créer une morale de l'amour capable de rivaliser avec la morale chrétienne : "Les circonstances, la situation de classe des seigneurs et chevaliers ont fait un beau succès à cette forme courtoise de l'amour païen déguisé sous des atours chrétiens ${ }^{25}$ ». Gautier a besoin de purifier tout cela et il veut des chansons débarrassées de cette immoralité ou de cette passion sacralisée. Il l'exprime avec ces jeux de rimes équivoquées dont il a le secret. :

\section{...Canter vos veil deus chançonnetes.}

Mout volentiers chant chançons netes ...(I, Pr 2, t. 1, p. 20, v. 7-8.)

Célébrer Notre-Dame comme la dame courtoise, ce n'est pas seulement imiter un modèle profane, mais retrouver, "récupérer ", l'origine véritable de cette divinisation littéraire de la femme aimée (Marie, elle, n'est pas une déesse) et de la sacralisation de l'amour, appelées à une grande postérité ${ }^{26}$.

Dans la littérature courtoise, narrative ou lyrique, la dame est bien souvent la cause de la prouesse, la destinataire et l'inspiratrice du poème. Dans les miracles, l'acte poétique est aussi un service rendu à la Dame immaculée. Il est prière et offrande. Grâce à lui, son nom, et celui de tous ses futurs lecteurs, seront écrits au grand livre de vie (t. 1, p. 22, v. 52-56). Marie est aussi le véritable coauteur du livre. C'est elle qui est chargée de purifier sa langue pour lui permettre de créer un œuvre digne de lui être présentée. Gautier utilise l'audacieuse image de la lime qui doit tout nettoyer dans son œuvre :

\section{La mere Dieu qui est la lime \\ Qui tout escure et tout eslime, \\ Escurer daint et eslimer, \\ La langue Gautier de Coinsi, \\ Qui por s'amor commence einsi. (t. 1, p. 19, v. 325-330)}

Marie, dans les miracles, présente à plusieurs reprises un livre à ses serviteurs (par exemple, t. 3, p.14-15). Symboliquement il faut y voir aussi l'œuvre littéraire offerte à son écrivain. Marie est la source de la grâce, de la courtoisie véritable et de la poésie, une poésie terrestre et céleste, humaine et divine à la fois. Rien d'étonnant puisqu'en elle le Verbe s'est fait chair. L'art religieux, tel qu'il apparaît à l'époque des cathédrales, est lié à une théologie de l'Incarnation. Dieu s'est fait homme en une femme, il a pris chair en elle. Le culte marial en découle. On souligne l'humanité du Christ. Georges Duby oppose cette théologie au dualisme cathare qui naît à cette époque : «Au second tiers du XII ${ }^{\mathrm{e}}$ siècle, ce dualisme spontané prit plus de consistance et s'érigea en véritable système doctrinal. [...] Cathare, ce mot apparait pour la première fois en 1163 appliqué aux sectateurs de ces croyances... Il ne s'agissait plus

\footnotetext{
${ }^{23}$ A. DRZEWICKA, op. cit. , p. 45. G. GROS reconnaît l'importance des travaux d'A. DRZEWICKA qui rétablit « une interprétation équilibrée » des rapports entre la poésie pieuse et la poésie profane. La première étant placée, toujours, «sous la tutelle » de la seconde. A notre avis, A. Drzewicka va plus loin encore, elle fait pencher la balance de l'autre côté (Perspectives Médiévales, p. 236.).

${ }^{24}$ P.-Y. BADEL, Introduction à la vie littéraire du Moyen Age, Paris, Bordas, 1969, p. 38.

${ }^{25}$ Y. LEFÈVRE, L'amour c'est le Paradis, commentaire de la chanson IX de Guillaume d'Aquitaine, Mélanges de littérature du Moyen Age, Bordeaux, éd. Brière, 1991, p. 173-174.

${ }^{26}$ Cf. par exemple, N. BOULESTREAU, La Poésie de Paul Eluard, Paris, éd. Klincksieck, 1985, p. 151.
} 
d'une déviation mais d'un autre dogme. [...] On entrevoit cette doctrine opposant un dieu bon à un dieu du Mal, un dieu de la lumière et de l'esprit, à un dieu de l'ombre et de la chair, dans une lutte à armes égales où se joue le sort du monde ${ }^{27}$. » Dans le christianisme, au contraire, la création est sortie bonne des mains de Dieu. «La matière participe à la splendeur de Dieu ; elle le glorifie ; elle conduit à le connaître ${ }^{28}$. " L'homme participe à cette création qui n'est pas achevée. Le travail de l'artisan et de l'artiste collaborent à l'œuvre de Dieu et le célèbrent. L'art des cathédrales est fondé sur cette esthétique. La pierre, le bois, l'or, travaillés par l'artiste disent la beauté de Dieu, qui est lumière. Cet art est aussi un art du trésor. Rien n'est trop beau pour louer Dieu. Les mots sont des choses précieuses (on peut les limer comme des joyaux, nous dit Gautier). La langue est aussi une matière que travaille et que cisèle le poète. Son travail d'écriture, d'une grande virtuosité, se rattache à cette esthétique du trésor. Le corps est impliqué dans l'acte de parole ${ }^{29}$. L'œuvre littéraire est consacrée à Notre-Dame, comme le sont la plupart des cathédrales ${ }^{30}$. Il est possible de chanter Marie et à travers elle de chanter Dieu, et cela en utilisant des codes formels et thématiques propres à une culture et à une époque données, quitte à en infléchir le sens. C'est elle qu'il chante, c'est elle qui chante en lui puisque, comme il le dit plaisamment dans un de ces jeux de mots dont il est spécialiste :

Ades fuisse ses escrivainz

Mais mout sui quant escri vains.

(t. 2, p. 92, v. 2301-2302)

Jean-Louis, Gabriel, BENOIT, université de Bretagne-Sud

\footnotetext{
${ }^{27}$ G. DUBY, Le Moyen Age, L'Europe des cathédrales, 1140-1280, Genève, 1966, p. 72.

${ }^{28}$ Ibidem, p . 129.

${ }^{29}$ CF. J.-L. BENOIT, Nourritures terrestres, célestes et poétiques dans les miracles de Nostre Dame de Gautier de Coinci, Cahiers de Recherches Médiévales, 2000, n 7, p. 213-225. Voir aussi M. C. POUCHELLE, Mots fluides et vertiges : les fêtes orales de la mystique chez Gautier de Coinci, Annales E.S.C., 1987, p. 1219.

${ }^{30} \mathrm{Il}$ y a une littérature des cathédrales qui s'inscrit dans l'art des cathédrales. Les miracles en font partie.
} 